\title{
Large-scale volumetric flow measurement in a pure thermal plume by dense tracking of helium-filled soap bubbles
}

\author{
Florian Huhn ${ }^{1}(0) \cdot$ Daniel Schanz $^{1} \cdot$ Sebastian Gesemann $^{1} \cdot$ Uwe Dierksheide $^{2}$. \\ Remco van de Meerendonk ${ }^{3} \cdot$ Andreas Schröder $^{1}$
}

\begin{abstract}
We present a spatially and temporally highly resolved flow measurement covering a large volume $\left(\sim 0.6 \mathrm{~m}^{3}\right)$ in a pure thermal plume in air. The thermal plume develops above an extended heat source and is characterized by moderate velocities $(U \sim 0.35 \mathrm{~m} / \mathrm{s})$ with a Reynolds number of $\operatorname{Re} \sim 500$ and a Rayleigh number of $\operatorname{Ra} \sim 10^{6}$. We demonstrate the requirements and capabilities of the measurement equipment and the particle tracking approach to be able to probe measurement volumes up to and beyond one cubic meter. The use of large tracer particles $(300 \mu \mathrm{m})$, helium-filled soap bubbles (HFSBs), is crucial and yields high particle image quality over large-volume depths when illuminated with arrays of pulsed high-power LEDs. The experimental limitations of the HFSBs-their limited lifetime and their intensity loss over time-are quantified. The HFSBs' uniform particle images allows an accurate reconstruction of the flow using Shake-The-Box particle tracking with high particle concentrations up to 0.1 particles per pixel. This enables tracking of up to 275,000 HFSBs simultaneously. After interpolating the scattered data onto a regular grid with a Navier-Stokes regularization, the velocity
\end{abstract}

Electronic supplementary material The online version of this article (doi:10.1007/s00348-017-2390-2) contains supplementary material, which is available to authorized users.

2) Florian Huhn

florian.huhn@dlr.de

1 Institute of Aerodynamics and Flow Technology, Department of Experimental Methods, German Aerospace Center (DLR), Bunsenstr. 10. Göttingen. Germany

2 LaVision GmbH, Anna-Vandenhoeck-Ring 19, Göttingen, Germany

3 Department of Aerospace Engineering, Delft University of Technology. Kluyverweg 1. Delft. The Netherlands field of the thermal plume reveals a multitude of vortices with a smooth temporal evolution and a remarkable coherence in time (see animation, supplementary data). Acceleration fields are also derived from interpolated particle tracks and complement the flow measurement. Additionally, the flow map, the basis of a large class of Lagrangian coherent structures, is computed directly from observed particle tracks. We show entrainment regions and coherent vortices of the thermal plume in the flow map and compute fields of the finite-time Lyapunov exponent.

\section{Introduction}

Time-resolved volumetric flow measurements, using methods such as tomo-PIV (Elsinga et al. 2006), 3D PTV (Maas et al. 1993; Malik et al. 1993) or Shake The Box (Schanz et al. 2013b, 2016a), are typically restricted to relatively small volume sizes of the order of $\leq 200 \mathrm{~cm}^{3}$ (Scarano et al. 2015). This limitation stems from the small size of commonly used seeding material, to accurately follow the fiow (diameter range around $\sim 1 \mu \mathrm{m}$ in air, $10-50 \mu \mathrm{m}$ in water). Currently available high-repetition rate laser systems, which are typically used as a light source, do not provide enough intensity to allow for illumination of larger volumes, even for the larger particles used in water experiments. However, seeding particles whose density approaches that of the medium can be of larger size. while still accurately being able to follow the flow (Melling 1997). In line with this thought, neutrally buoyant helium-filled soap bubbles (HFSBs) have been used in air to allow for large-scale flow measurements in the laboratory. Applications range from traceline visualizations (Pounder 1956) over largescale 2D-PIV measurements (Müller et al. 2000; Bosbach et al. 2009) to three-dimensional tracking of single bubbles 